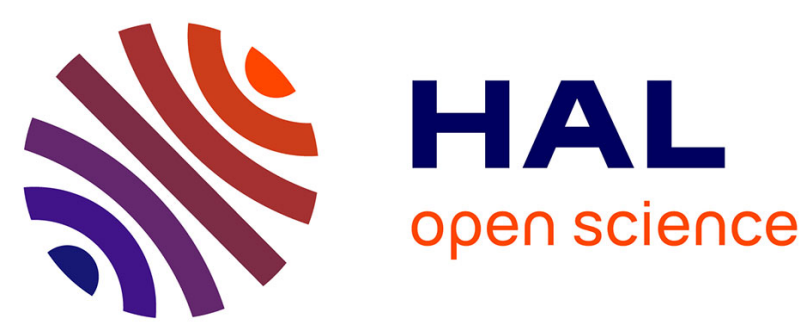

\title{
La détection automatique
}

Michel Laug

\section{To cite this version:}

Michel Laug. La détection automatique. Revue forestière française, 1975, 27 (S), pp.352-357. 10.4267/2042/20993 . hal-03396415

\section{HAL Id: hal-03396415 https://hal.science/hal-03396415}

Submitted on 22 Oct 2021

HAL is a multi-disciplinary open access archive for the deposit and dissemination of scientific research documents, whether they are published or not. The documents may come from teaching and research institutions in France or abroad, or from public or private research centers.
L'archive ouverte pluridisciplinaire HAL, est destinée au dépôt et à la diffusion de documents scientifiques de niveau recherche, publiés ou non, émanant des établissements d'enseignement et de recherche français ou étrangers, des laboratoires publics ou privés. 


\section{La détection automatique}

S'il est vrai que le guetteur humain est irremplaçable à divers niveaux de la surveillance des forêts en raison de ses facultés d'interprétation, de décision, d'intervention, il ne peut pas pour autant assurer seul dans de bonnes conditions toute la responsabilité de l'alerte. Il se pose en effet des problèmes de fiabilité (fatigue, champ d'observation limité), de densité d'occupation des forêts (effets psychologiques sur un guetteur isolé, insécurité des points peu accessibles), de permanence de la veille (qui implique des effectifs élevés).

Ces problèmes, assez facilement résolus dans une forêt de rapport comme les Landes, qui bénéficient en plus d'un relief plat, sont fortement ressentis dans d'autres forêts, particulièrement celles du sudest.

II est donc important de réfléchir aux solutions techniques susceptibles de réduire les délais d'alerte en toutes circonstances. Nos travaux ont pour but de préciser la nature de quelques solutions possibles et leur domaine d'utilisation.

\section{LES BESOINS}

Les services des forêts et de la protection civile peuvent être amenés à définir des besoins variés en matière de détection, par exemple :

- déterminer en permanence les conditions météorologiques propices à un départ de feu : un système répondant à ce souci a été mis en service par la NASA et la «California Division of Forestry 》;

- localiser à travers la fumée le foyer d’un incendie qui a déjà pris une certaine extension : un appareil américain utilisé sur avion et sur satellite effectue cette recherche à l'aide d'un radiomètre infrarouge sensible à la longueur d'onde de $3,4 \mu$, où la flamme émet un rayonnement intense et où la fumée est transparente;

- déceler et localiser un début d'incendie dans les meilleurs délais.

Un dialogue préliminaire avec les forestiers français nous a orientés vers ce dernier objectif. De plus, nous nous sommes imposé les spécifications suivantes :

- longue portée, de manière à réduire la densité de stations, non tellement pour diminuer le coût (le prix unitaire risquant d’être plus élevé) que pour en faciliter la surveillance et la maintenance ;

- grande autonomie, donc faible consommation;

- aptitude à détecter les fumées, étant donné que les flammes se manifestent rarement à distance dès les premiers instants.

\section{L'APPAREIL EXPÉRIMENTAL : UN RADIOMĖTRE INFRAROUGE}

L'impératif «longue portée » nous a fait choisir un moyen optique comme solution de base, malgré tout l'intérêt des capteurs de proximité pour certaines applications spécifiques (des «renifleurs» ont été proposés et essayés avec succès dans la forêt de Fontainebleau* ${ }^{*}$ ). De même, voulant consommer peu d'énergie, on exclut les méthodes actives, qui nécessitent des sources de lumière (projecteurs et lasers), au moins en régime de veille permanente. On s'intéresse donc principalement aux détecteurs optiques passifs.

- Voir « Figaro» du 19 mai 1972. 


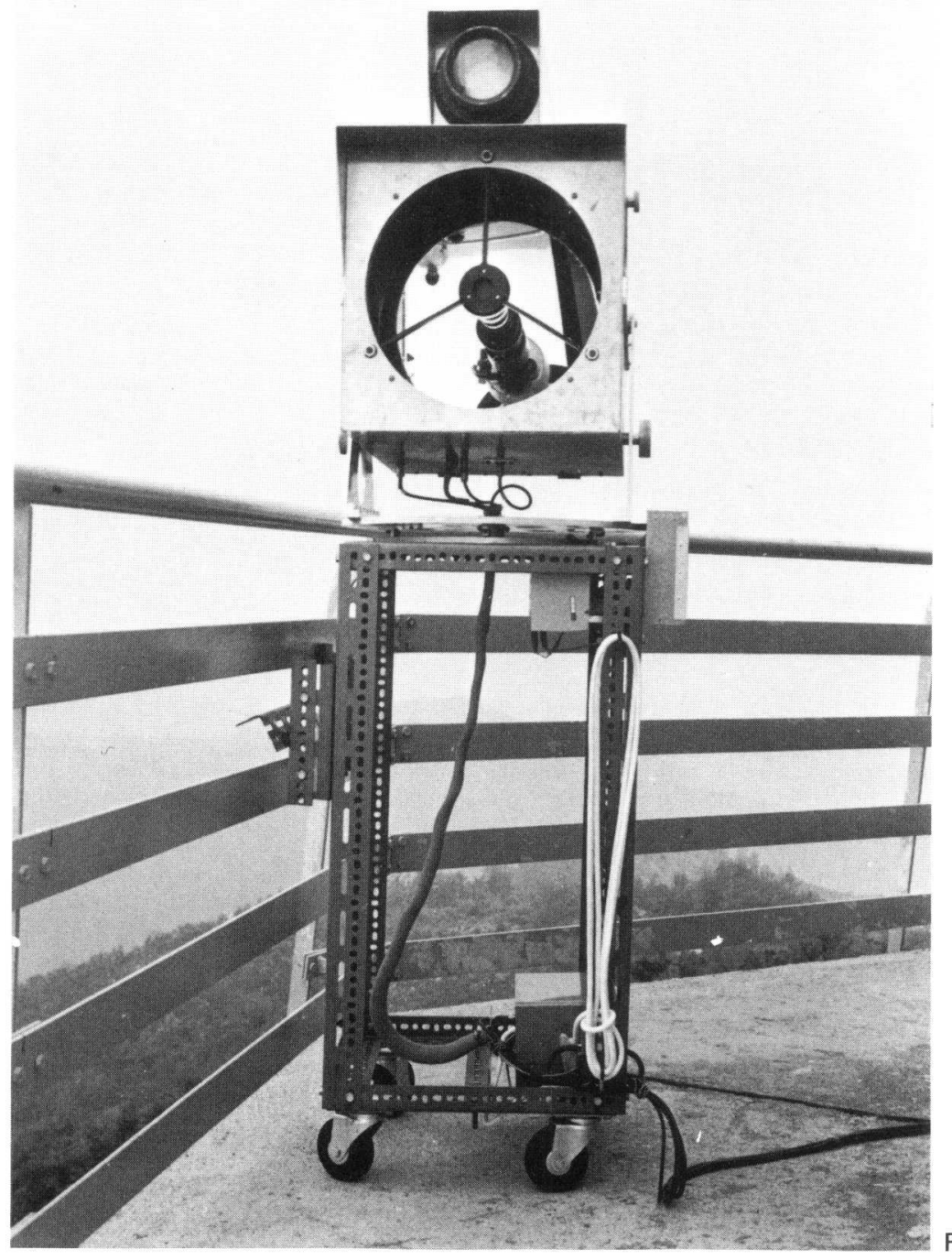

Figure $n^{0} 1$

Photo $X$

Ces détecteurs répondent aux variations de rayonnement qui résultent de la présence des fumées : rayonnement propre des particules et gaz chauds, qui s'ajoute au rayonnement ambiant, accroissement (par diffusion) ou diminution (par absorption) du rayonnement ambiant.

Le premier phénomène est prépondérant dans l'infrarouge, tandis que le deuxième est prépondérant dans le visible. Comme les élévations brusques de températures sont plus rares que les causes de perturbation du rayonnement ambiant, on peut s'attendre à une interprétation plus facile en détectant le rayonnement infrarouge. Toutefois, inspirés par les succès des détecteurs de rayonnement visible que sont les guetteurs humains, nous n'avons pas exclu immédiatement cette gamme de longueurs d'onde $(0,4 \mu-0,8 \mu)$.

Le détecteur choisi est le bolomètre, qui a le mérite de répondre à tous les rayonnements et de fonctionner à température ordinaire (une installation cryogénique serait en effet un lourd handicap). Un bolomètre est un appareil qui s'échauffe quand il reçoit un rayonnement quelconque et dont on mesure précisément l'élévation de température, soit grâce à sa variation de résistance électrique (si la surface sensible est une thermistance), soit à l'aide d'un thermocouple.

Le rayonnement est reçu sur un miroir de manière à en capter suffisamment, puis converge vers le bolomètre. A l'entrée de celui-ci, on place différents filtres, selon la gamme de longueurs d'onde qu'on désire étudier.

Le radiomètre infrarouge ainsi constitué, complété par une électronique amplifiant le signal électrique de sortie du bolomètre, muni d'une lunette permettant de connaître le champ visé par le détecteur, et monté sur une plateforme tournante, constitue l'appareil expérimental qui nous a permis de recueillir les données nécessaires à la définition d'un prototype (figure nº 1). 
Le choix des longueurs d'onde aurait dû résulter d'une analyse détaillée des gaz et des particules solides contenus dans les fumées. N'ayant pas eu connaissance d'une telle étude, nous avons retenu à priori, les gammes suivantes :

- visible $(0,4-0,8 \mu)$, avec une division en sous-gammes ;

- autour de 3,4 $\mu$, bande d'émission maximale à la température de la flamme ;

- autour de 6,3 $\mu$, bande d'émission de la vapeur d'eau présumée présente dans la fumée;

- autour de $10 \mu$, bande d'émission maximale à la température voisine de l'ambiante pour les particules solides (il se trouve qu'aucun gaz connu et susceptible d'entrer dans la composition des fumées n'émet dans cette bande);

- autour de $15 \mu$, bande d'émission maximale du dioxyde de carbone $\left(\mathrm{CO}_{2}\right)$.

La vitesse de rotation de la plateforme est limitée par le temps de réponse du bolomètre à environ un demi-tour en 30 secondes. Elle a été fixée à un demi-tour en 90 secondes.

L'appareil a été expérimenté deux fois dans la région de Gonfaron-Collobrières (Var) :

- en été 1973 sur des feux accidentels,

- au printemps 1974 sur des feux provoqués.

\section{CAMPAGNE 1973}

Le détecteur était situé sur la tour de Notre-Dame-des-Anges, qui est dotée d'un guetteur chaque été. N'étant pas conçu pour un service permanent, il n'était actionné qu'après identification d'un incendie par le guetteur ou pour des mesures diverses (nuages, émissions de dépôts d’ordures).

Cinq incendies se sont déclarés pendant cette période :

- à $5 \mathrm{~km}$ avec flamme à vue directe;

- à 4,5 km derrière une crête, d'où émergeait une fumée à peine perceptible à l'œil nu ;

- à $14 \mathrm{~km}$ derrière une crète, feu d'herbes et de broussailles;

- deux fois à plus de $30 \mathrm{~km}$.

La voie visible a donné des signaux très caractéristiques sur la flamme du premier incendie et semble avoir répondu aux fumées, mais d'une façon difficilement discernable au milieu de variations fréquentes et complexes. La voie $3,4 \mu$ n'a répondu qu’à la flamme.

Les voies $6,3 \mu$ et $15 \mu$ n'ont rien donné, soit en raison de concentrations insuffisantes de vapeur d'eau et de $\mathrm{CO}_{2}$, soit en raison de l'absorption de ce rayonnement entre la fumée et le détecteur, puisquel'atmosphère contient précisément ces mêmes gaz.

La voie $10 \mu$ a donné dans les trois premiers cas un signal exploitable à chaque passage du détecteur dans la direction de la fumée. Même à $14 \mathrm{~km}$, le signal se caractérise par un couple intensité - temps de montée qui le distingue nettement des autres fluctuations de rayonnement.

Les feux situés à plus de $30 \mathrm{~km}$ n'ont été observés sur aucune voie.

Précisons en outre que l'appareil fonctionnait sur le secteur, disponible à Notre-Dame-des-Anges et utile dans cette première version, qui devait fournir une haute tension au bolomètre à thermistance.

\section{CAMPAGNE 1974}

Les résultats de la campagne 1973 nous ont conduit à reprendre des expériences avec un appareil limité à la voie $10 \mu$, plus précisément équipé d'un filtre de $8 \mu$ à $12 \mu$, gamme correspondant à la « fenêtre atmosphérique » (I'atmosphère ne contient aucun gaz absorbant ce rayonnement). En effet, tout rayonnement extérieur à cette gamme constitue plus probablement un bruit qu'un signal utile.

En outre, nous avons utilisé un bolomètre à thermocouple, plus sensible et alimenté en basse tension : l'appareil, fonctionnant sur accumulateurs ou piles, devenait ainsi autonome.

Le détecteur, installé sur un camion, se déplaçait en différents points pour observer des feux allumés volontairement en un point fixe. Le foyer était constitué par des rémanents de même essence que les arbres de la forêt et situé dans une tranchée pare-feu de manière à soustraire les flammes aux vues directes depuis les postes d'observation. La figure $n^{\circ} 2$ donne une idée de son importance. Un début d'incendie et son observation dans des conditions réalistes étaient ainsi bien simulés.

Une liaison radio était assurée entre la station fixe, chargée d'allumer les feux, et la station mobile, chargée de la détection. La séquence des opérations était la suivante :

- Mise en route du détecteur, effectuant un balayage va-et-vient (dont l'inversion était commandée par un commutateur manuel) autour de la direction de la tranchée. 


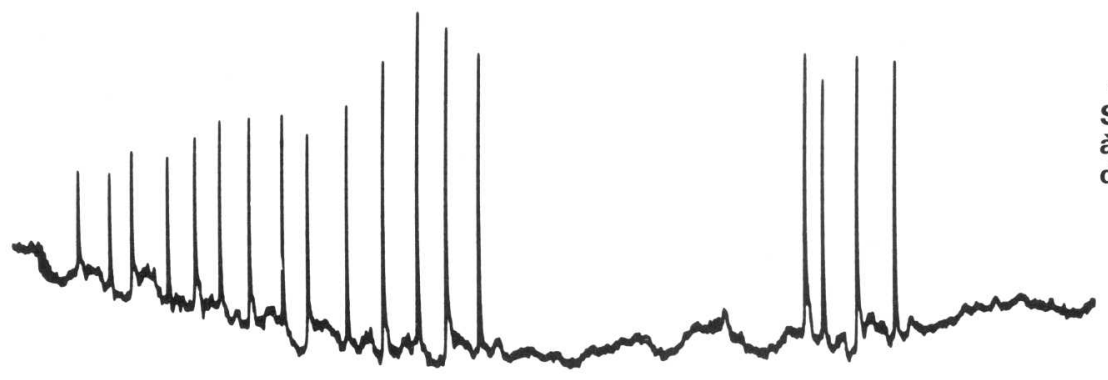

Figure $n^{\circ} 2$

Signal obtenu

à $9 \mathrm{~km}$, en cours de combustion.

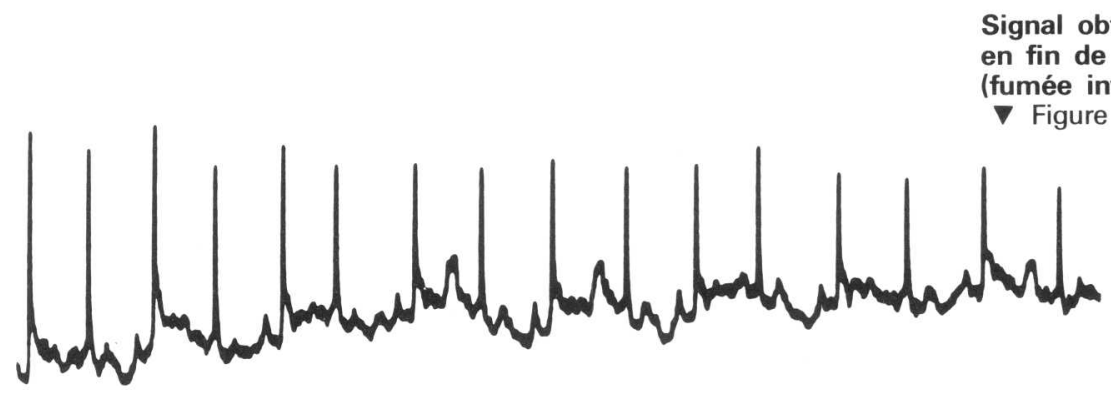

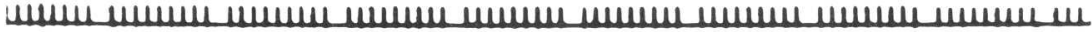

- Émission d'un signal "prêt» de la station mobile vers la station fixe.

- Allumage du feu.

- Émission immédiate d'un signal «feu» de la station fixe vers la station mobile.

Quatre feux successifs ont été allumés, la station mobile se situant respectivement à une distance de $3,5,9$ et $12 \mathrm{~km}$ à vol d'oiseau.

Le rayonnement a été capté les quatre fois par le radiomètre infrarouge, pratiquement en simultanéité avec le signal «feu ». La sortie du bolomètre était connectée à un enregistreur. Les résultats obtenus lors du troisième essai (à $9 \mathrm{~km}$ ) sont reproduits ci-dessous : le premier signal (figure $\mathrm{n}^{\circ} 2$ ) était obtenu en cours de combustion, le deuxième (figure $n^{\circ} 3$ ) en fin de combustion, alors que les fumées n'étaient plus visibles par l'homme. Chaque passage sur la fumée a donné l'une des impulsions reconnaissables sur les figures. Le rapport signal/bruit est supérieur à 50 en cours de combustion, de l'ordre de 20 en fin de combustion.

De même, aucune fumée n'était visible par l'homme au cours du quatrième essai en raison de la distance $(12 \mathrm{~km})$ et d'une légère brume. Le radiomètre infrarouge y a encore accusé un rapport signal/bruit de 30 . 


\section{BILAN DES EXPÉRIENCES}

Les deux campagnes décrites ci-dessus ont montré qu'il est d'ores et déjà possible de détecter un incendie jusqu'à une distance de l'ordre de $15 \mathrm{~km}$ et de déclencher une alarme moins d'une minute après sa naissance. Cette limite résulte du temps de rotation du radiomètre pour un tour complet, dans le cas où toutes les directions doivent être surveillées (angle de $360^{\circ}$ en azimut).

Le champ observé par le radiomètre fixe est de 1 degré, valeur destinée, en ce qui concerne l'azimut, à garantir un bon rapport signal/bruit. Cet angle pourrait être augmenté, mais au détriment de la portée. Malheureusement pour un appareil à champ circulaire, le site surveillé n'est que de 1 degré également. II faudrait donc effectuer par exemple 10 balayages successifs à dix sites différents pour surveiller un secteur de 10 degrés en site : le délai d'alerte maximal passerait alors à 10 minutes (toujours pour une portée de $15 \mathrm{~km}$ ).

Le coût des composants pour un modèle unique est de $8000 \mathrm{~F}$. Les frais d'étude et de montage étant probablement un peu supérieurs à l'abaissement des coûts de composants achetés en série, le prix de revient définitif serait, avec les spécifications obtenues lors des essais, un peu inférieur à $10000 \mathrm{~F}$.

Une question importante, qui n'a pas encore fait l'objet d'expériences poussées, est celle des fausses alarmes. Tout au plus avons-nous observé quelques fumées d'un dépôt d'ordures situé à $5 \mathrm{~km}$, qui n'ont donné aucun signal, et quelques transitions de nuage à ciel clair, qui ont donné un signal beaucoup plus faible que les incendies. Mais il resterait, avant de conclure que la discrimination des incendies sera aisée, à multiplier les expériences sur de nombreuses sources de rayonnement à différentes distances. Le soleil à vue directe donne incontestablement une fausse alarme, mais à heure connue (le matin et le soir) et dans une direction connue : il est donc facile d'inhiber le signal correspondant.

Dans l'état actuel, malgré un effort modique à cet égard, la fiabilité est déjà convenable : les parties optique et électronique de l'appareil ne devraient pas subir plus d'une panne par an en fonctionnement permanent; la mécanique ne devrait pas poser beaucoup de problèmes.

L'appareil est entièrement autonome pendant 50 heures avec deux piles de 12 volts.

\section{PERSPECTIVES D'AVENIR}

L'étude doit être poursuivie selon deux perspectives différentes.

\section{Aide au guetteur}

Le système comprendrait par exemple :

- un détecteur principal balayant les 360 degrés avec la portée maximale, placé sur la même tour que le guetteur : son but serait d'assurer une veille permanente sur tout l'horizon et d'étendre la la surveillance aux périodes de brume;

- plusieurs détecteurs situés sur les crêtes des contrepentes, surveillant les zones cachées au guetteur : leur portée serait plus réduite, ainsi que leur champ d'observation, ce qui permettrait d'accroître leur autonomie et de réduire leur coût.

Chaque détecteur fournirait au guetteur une alarme sonore et visuelle avec une indication sommaire de la direction.

Dans cette conception, les fausses alarmes ne seraient sans doute pas très graves, surtout si le guetteur a des moyens pour recouper ces informations. II serait alors facile de tendre rapidement vers des appareils opérationnels, assez semblables à l'appareil expérimental. Les améliorations essentielles à apporter seraient les suivantes :

- accroître l'autonomie, soit en plaçant plusieurs séries de deux piles en parallèle, soit en imaginant une autre source d'énergie pour la rotation (moteur à poids) et/ou pour l'électronique (batteries solaires):

- accroître l'angle de site du secteur surveillé, au moins pour le détecteur principal, sans pour autant augmenter exagérément le délai d'alerte; il faut pour cela demander le maximum à l'optique et seulement le complément à la mécanique,

- effectuer tout de même un traitement de signal sommaire, pour réduire les fausses alarmes,

- améliorer la fiabilité au stade de l'industrialisation,

- chercher un capteur à réponse plus rapide et corrélativement balayer plus vite, à la fois pour réduire le délai d'alerte et pour améliorer le rapport signal/bruit (en filtrant avec une fréquence de coupure plus élevée). 


\section{Réseau automatique}

Une étude sur papier nous permet de présenter une solution de réseau entièrement automatique, surveillant de grandes surfaces de forêts et transmettant l'alerte à un poste de commandement central après un traitement propre à ramener les taux de non-détection et de fausses alarmes à un niveau acceptable.

Faute de réalisation pratique à ce jour, nous avons préféré réserver la totalité de la place offerte dans cette revue à la description du capteur et de son expérimentation.

En outre, un tel système ne pourrait pas être rendu opérationnel à très court terme et les dépenses qu'il impliquerait semblent réduire l'intérêt de ces techniques dans l'esprit des utilisateurs.

\section{CONCLUSIONS}

Le Centre d'études et de recherches de l'École nationale supérieure de l'aéronautique et de l'espace a construit un appareil expérimental, qui a démontré la possibilité de détecter les incendies de forêts par voie optique et qui a présenté très rapidement des spécifications acceptables dans l'ensemble.

L'intérêt d'une telle solution semble évident si l'on considère le nombre d'incendies qui prennent chaque année des proportions dramatiques pour avoir été détectés trop tard, dans des situations où il serait exclu de multiplier le nombre de guetteurs.

Nous croyons avoir prouvé que la radiométrie infrarouge permettrait d'installer à court terme et sans investissements excessifs, des systèmes qui, mis à la disposition des guetteurs, accroîtraient considérablement leur efficacité.

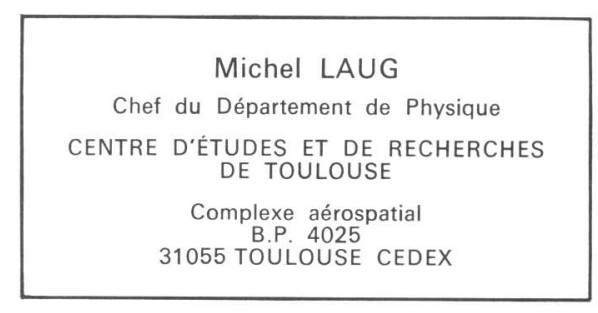

